\title{
Intestinal complications after chemotherapy for patients with unresected primary colorectal cancer and synchronous metastases
}

\author{
N C Tebbutt, A R Norman, D Cunningham, M E Hill, D Tait, J Oates, S Livingston, \\ J Andreyev
}

See end of article for authors' affiliations

Correspondence to: Professor D Cunningham Department of Medicine, Royal Marsden Hospital, Downs Road, Sutton, Surrey SM2 5PT, UK; david.cunningham@icr.ac.uk

Accepted for publication 15 October 2002

\begin{abstract}
Background: The role of palliative resection of the primary tumour in patients who present with metastatic colorectal cancer is unclear.

Aims: This study compared the incidence of major intestinal complications in such patients who received chemotherapy treatment with or without prior palliative resection of the primary fumour.

Patients: The incidence of intestinal obstruction, perforation, fistula formation, and gastrointestinal haemorrhage, and the requirement for abdominal radiotherapy in patients with metastatic colorectal cancer treated at a single institution over a 10 year period was determined.

Results: Eighty two patients received initial treatment with chemotherapy without resection of the primary tumour (unresected group) and 280 patients had undergone prior resection (resected group). In the unresected group, the incidence of peritonitis, fistula formation, and intestinal haemorrhage was $2.4 \%(95 \%$ confidence interval $(\mathrm{Cl}) 0.3-8.5 \%), 3.7 \%(95 \% \mathrm{Cl} 0.8-10.3 \%)$, and $3.7 \%(95 \% \mathrm{Cl} 0.8-$ $10.3 \%)$, respectively, and was not significantly different from the resected group. Intestinal obstruction affected $13.4 \%(95 \% \mathrm{Cl} 6.9-22.7 \%)$ of patients in the unresected group and $13.2 \% 195 \% \mathrm{Cl}$ $9.2-17.2 \%)$ of patients in the resected group. More patients in the unresected group required $\geqslant 3$ blood transfusions $(14.6 \% \vee 7.5 \% ; p=0.048)$ and abdominal radiotherapy $(18.3 \% \vee 9.6 \% ; p=0.03)$ than the resected group.

Conclusions: The incidence of major intestinal complications in patients with unresected colorectal cancer and synchronous metastases who receive initial treatment with chemotherapy is low. Chemotherapy may be successfully used as initial treatment for such patients with no increased risk of most major intestinal complications compared with patients who have undergone initial resection of the primary tumour.
\end{abstract}

$A^{n}$ proximately $25 \%$ of patients with colorectal cancer have metastatic disease at the time of first presentation. ${ }^{2}$ The principal goals of management in this setting are maintenance of quality of life and prolongation of survival. Patients who present with metastatic colorectal cancer, where the primary tumour has not been resected, pose a common problem for clinical management. In this situation, palliative resection of the primary tumour is frequently performed, with the aim of treating associated symptoms and preventing local complications such as intestinal obstruction, perforation, or major haemorrhage which have an adverse impact on quality of life. ${ }^{3}$ However, this surgical procedure itself carries a risk of morbidity and mortality and may not be the optimal method of controlling tumour related symptoms, particularly where these are due to metastatic disease. Furthermore, in those considered suitable for systemic chemotherapy, surgery may delay or prevent altogether the palliative benefits associated with such treatment.

The gastrointestinal unit at the Royal Marsden Hospital (RMH) has frequently used chemotherapy as the initial treatment for patients presenting with metastatic colorectal cancer and an unresected primary tumour. It was not possible to assess retrospectively the effects of this approach on symptoms or overall quality of life accurately. Therefore, in this study, the incidence of major intestinal complications such as obstruction, perforation, and haemorrhage, as well as requirement for abdominal radiotherapy, which were likely to be associated with a significant adverse effect on overall quality of life, were assessed in this group of patients. The study also aimed to estimate the potential benefits associated with palliative resection of the primary tumour before chemotherapy by assessing the incidence of intestinal complications in patients who had presented with metastatic disease but who had undergone initial palliative surgical resection of the primary tumour.

\section{PATIENTS AND METHODS Patients}

The gastrointestinal unit at RMH has maintained a prospective database of all patients participating in clinical trials since 1990. This database contains details of patient demographics, sites of metastatic disease based on computed tomography (CT) scan results, prior and subsequent surgical treatment, chemotherapy treatment, haematological and biochemical parameters, and survival. It was used to identify and obtain information on all patients who had presented to RMH with metastatic colorectal cancer and who had received chemotherapy in the context of a clinical trial. These patients were allocated to one of two groups: those in whom the primary tumour was left unresected before chemotherapy (unresected group) and those in whom the primary tumour was resected before chemotherapy (resected group).

Patients in the unresected group were required to have presented to RMH with metastatic colorectal cancer and to have

Abbreviations: CEA, carcinoembryonic antigen; $\mathrm{CT}$, computed tomography; 5-FU, 5- fluorouracil; HR, hazard ratio; Nd,YAG neodymium yttrium aluminium garnet; RMH, Royal Marsden Hospital. 
undergone no surgical procedure other than biopsy and/or endoscopic examinations. Patients who had undergone bypass or defunctioning surgery, but whose primary tumour was unresected, were thereby excluded. The unresected group included patients whose primary tumour was symptomatic. These symptoms included alteration of bowel frequency, rectal bleeding, and pain.

Patients in the resected group were required to have presented to RMH with metastatic colorectal cancer and to have undergone complete surgical resection of the primary tumour within three months prior to commencement of chemotherapy. As a result of variation in preoperative staging methods, the resected group contained patients in whom the diagnosis of metastatic disease was known prior to surgical resection as well as patients in whom the diagnosis of metastatic disease was discovered either at the time of surgery or immediately after surgery.

Primary tumours were classified as right sided tumours (arising in the caecum or ascending colon), residual colon tumours (arising at any site between the hepatic flexure and the sigmoid colon), and rectal tumours (arising in the rectum). This classification was used as it was anticipated that intestinal bleeding would be more likely to occur with right sided tumours whereas large intestinal obstruction would be more likely with tumours in the residual colon or rectum. The presence of peritoneal or omental metastases was determined based on histopathological evidence at surgery and/or radiological evidence on CT scan.

\section{Assessment of intestinal complications}

The incidence of intestinal complications during the period of follow up was determined by a combination of individual case note review and analysis of electronic bloodbank records.

Intestinal obstruction was defined on the basis of clinical features (abdominal distension, vomiting, constipation) in conjunction with a plain abdominal radiograph demonstrating at least one dilated bowel loop in order to exclude other causes of abdominal distension such as ascites or constipation. The radiological reports frequently did not distinguish between large and small intestinal obstruction, such that cases of documented intestinal obstruction could not be accurately subclassified. In addition, for the purposes of this analysis, any patient from the unresected group who required subsequent intestinal bypass or defunctioning surgery was classified as having developed intestinal obstruction as these procedures were invariably performed in order to avert incipient intestinal obstruction.

Peritonitis was defined on the basis of clinical features (abdominal tenderness, rebound tenderness, guarding, and absent bowel sounds). The presence of fistulae (enterocutaneous, enterovesical, or enterovaginal) was defined on the basis of clinical features with appropriate confirmatory radiological imaging as required.

Episodes of major gastrointestinal haemorrhage were defined as hospital admissions for haematemesis or melaena. The severity of occult gastrointestinal haemorrhage was estimated based on transfusion requirements during the period of active follow up, excluding perioperative transfusions. The number of admissions for transfusion was defined as the number of days on which a patient received blood. This frequently coincided with an admission for chemotherapy and thus did not often require separate admission.

Abdominal radiotherapy was defined as radiotherapy to target lesions between the diaphragm and pelvis.

\section{Statistical analysis}

Statistical analysis was performed using the statistical package SPSS for Windows version 10.1. Categorical data were examined using the $\chi^{2}$ test, with Fisher's exact test used where appropriate. Survival and time to events were calculated using the Kaplan-Meier method ${ }^{5}$ and were measured from the date of commencement of chemotherapy for all patients. The logrank test ${ }^{6}$ was used to test differences between groups. Multivariate Cox regression or logistic regression analysis ${ }^{7}$ was used to identify prognostic groups that influenced survival, intestinal obstruction, major intestinal haemorrhage, and high transfusion requirements. Factors included in these analyses were resection status, age, sex, site of tumour and performance status, pretreatment haemoglobin, alkaline phosphatase, albumin, and carcinoembryonic antigen (CEA). A p value less than 0.05 was considered significant.

\section{RESULTS}

\section{Patients and treatment}

Between I January 1990 and I January 2000, 410 patients had presented to RMH with metastatic colorectal cancer and commenced firstline chemotherapy on a trial protocol. The drug regimens used were based on 5 -fluorouracil $(5-\mathrm{FU})$ in $80 \%$ of cases, and in the remaining cases were based on raltitrexed, capecitabine, and uracil tegafur. Results from these studies have been reported individually. ${ }^{8-12}$

Forty one patients were ineligible for this analysis because they had undergone a surgical bypass or defunctioning procedure and/or had undergone incomplete resection of the primary tumour, did not have metastatic disease, or had not presented with de novo metastatic disease. Case notes were missing in a further seven cases. Of the remaining 362 patients, 82 had an unresected primary tumour and 280 had undergone prior surgical resection.

Pretreatment characteristics of patients in the unresected and resected groups are shown in table 1. The unresected group had a statistically significantly worse performance status, lower serum albumin, and higher alkaline phosphatase and CEA, suggesting a worse overall prognosis. There were similar proportions of patients in each group with right sided residual colon or rectal primary tumours and with peritoneal and/or omental metastases, which were factors postulated to be associated with the risk of intestinal obstruction.

Follow up at RMH was maintained until the patient died, became unfit for further chemotherapy, or declined further chemotherapy. Median follow up was 19 months for the unresected group and 30 months for the resected group. Patients in the unresected group received, on average, 1.6 lines of palliative chemotherapy (range 1-3) compared with patients in the resected group who received on average 1.9 lines of treatment (range 1-6). Following chemotherapy treatment, metastasectomy together with resection of the primary tumour was performed in one patient in the unresected group and metastasectomy alone was performed in five patients in the resected group.

\section{Intestinal complications}

In the unresected group, 11 patients developed intestinal obstruction (13.4\%, 95\% confidence interval (CI) 6.9-22.7) (table 2). Surgical intervention occurred in eight cases and this included three patients classified as having obstruction who underwent surgical diversion in order to avert admission with acute large bowel obstruction. The remaining three cases were treated with conservative management. One patient in this group suffered more than one episode of obstruction.

In the resected group, 37 patients developed intestinal obstruction (13.2\%, 95\% CI 9.2-17.2). Surgical intervention occurred in 14 cases and among these cases there was evidence of obstruction due to recurrent intra-abdominal tumour in nine cases and due to intestinal adhesions in five. The remaining patients were treated conservatively. Five patients suffered more than one episode of obstruction.

There was no significant difference between the unresected and resected groups with respect to time to develop obstruction (fig 1A). However, the presence of peritoneal or 
Table 1 Baseline demographics of the unresected and resected groups

\begin{tabular}{|c|c|c|c|c|c|}
\hline & \multicolumn{2}{|c|}{ Unresected group } & \multicolumn{2}{|c|}{ Resected group } & \multirow[b]{2}{*}{$\mathrm{p}$ Value } \\
\hline & No & $\%$ & No & $\%$ & \\
\hline Total & 82 & 100 & 280 & 100 & \\
\hline $\begin{array}{l}\text { Median time from diagnosis to } \\
\text { chemotherapy (days) }\end{array}$ & 26 & & 52 & & \\
\hline \multicolumn{6}{|l|}{ Sex } \\
\hline Male & 60 & 73 & 169 & 60 & \\
\hline Female & 22 & 27 & 111 & 40 & 0.03 \\
\hline \multicolumn{6}{|l|}{ Age $(y)$} \\
\hline Median (range) & \multicolumn{2}{|c|}{$59(19-80)$} & \multicolumn{2}{|c|}{$62(30-82)$} & \\
\hline \multicolumn{6}{|c|}{ PS at commencement of chemotherapy } \\
\hline $0-1$ & 51 & 62 & 225 & 80 & \\
\hline$\geqslant 2$ & 31 & 38 & 55 & 20 & 0.001 \\
\hline \multicolumn{6}{|l|}{ Site of primary tumour } \\
\hline Right sided & 13 & 16 & 69 & 25 & \\
\hline Residual colon & 31 & 38 & 119 & 43 & \\
\hline Rectum & 38 & 46 & 92 & 33 & 0.06 \\
\hline \multicolumn{6}{|l|}{ Sites of metastases } \\
\hline Peritoneal/omental & 11 & 13 & 56 & 20 & \\
\hline Non-peritoneal/omental & 71 & 87 & 224 & 80 & 0.18 \\
\hline \multicolumn{6}{|l|}{ Biochemical parameters } \\
\hline Mean serum alk phosph (U/I) & 329 & & 179 & & $<0.001$ \\
\hline Mean serum albumin (g/l) & 34.5 & & 38.7 & & $<0.001$ \\
\hline Mean CEA ( $\mu \mathrm{g} / \mathrm{l})$ & 3139 & & 809 & & 0.006 \\
\hline
\end{tabular}

Table 2 Incidence of intestinal complications in the unresected and resected groups.

\begin{tabular}{|c|c|c|c|c|c|c|c|}
\hline & \multicolumn{3}{|c|}{ Unresected group } & \multicolumn{3}{|c|}{ Resected group } & \multirow[b]{2}{*}{$\mathrm{p}$ Value } \\
\hline & No & $\%$ & $95 \% \mathrm{Cl}$ & No & $\%$ & $95 \% \mathrm{Cl}$ & \\
\hline Intestinal obstruction & 11 & 13.4 & $6.9-22.7$ & 37 & 13.2 & $9.2-17.2$ & 0.96 \\
\hline Peritonitis & 2 & 2.4 & $0.3-8.5$ & 1 & 0.4 & $0-1.1$ & 0.13 \\
\hline Fistula & 3 & 3.7 & $0.8-10.3$ & 5 & 1.8 & $0.2-3.3$ & 0.39 \\
\hline Admission for intestinal haemorrhage & 3 & 3.7 & $0.8-10.3$ & 11 & 3.9 & $1.7-6.2$ & 1.0 \\
\hline Total blood transfusion $\geqslant 5$ units & 17 & 20.7 & $12.6-31.1$ & 33 & 11.8 & $8.0-15.6$ & 0.039 \\
\hline$\geqslant 3$ transfusions & 12 & 14.6 & $7.8-24.2$ & 21 & 7.5 & $4.4-10.6$ & 0.048 \\
\hline Intestinal radiotherapy & 15 & 18.3 & $10.6-28.4$ & 27 & 9.6 & $6.2-13.1$ & 0.031 \\
\hline
\end{tabular}
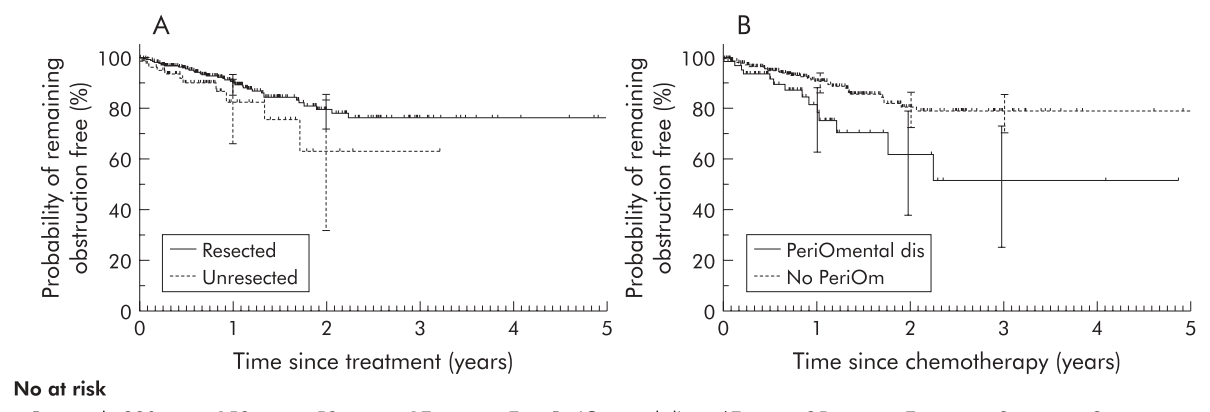

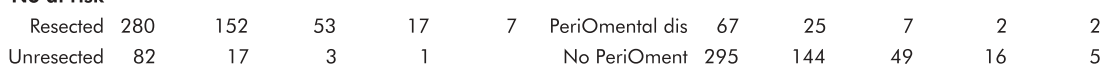

Figure 1 (A) Comparison of time until the development of intestinal obstruction in the unresected and resected groups $(p=0.11)$.

(B) Comparison of time until the development of intestinal obstruction in patients with (PeriOmental) and without (No PeriOm) peritoneal or omental metastases $(p=0.006)$.

omental metastases was associated with an increased risk of early intestinal obstruction (fig $1 B)(p=0.006)$. In order to identify factors associated with intestinal obstruction, while controlling for the differences between groups, multivariate Cox regression analysis was performed. This showed that the presence of peritoneal or omental metastases (hazard ratio (HR) $1.98,95 \%$ CI $1.03-3.82 ; \mathrm{p}=0.04$ ) and younger age (HR $0.96,95 \%$ CI $0.93-0.98 ; p=0.001$ ) were the most significant factors associated with increased risk of intestinal obstruction (table 3). Neither resection status $(p=0.06)$ nor the site of the primary tumour $(\mathrm{p}=0.21)$ was associated with an increased risk of intestinal obstruction.

Two patients in the unresected group $(2.4 \%, 95 \%$ CI $0.3-8.5 \%)$ and one patient in the resected group $(0.4 \%, 95 \% \mathrm{CI}$ $0-1.1 \%$ ) developed clinical signs of peritonitis. All three patients were judged to be unfit for surgical treatment of the peritonitis based on their clinical condition. Three patients in the unresected group $(3.7 \%, 95 \%$ CI $0.8-10.3 \%)$ and five patients in the resected group developed fistulae (1.8\%, 95\% CI $0.2-3.3 \%)$. There was no significant difference in the incidence of peritonitis or fistulae between the unresected and resected groups. 
Table 3 Cox regression analysis of intestinal obstruction

\begin{tabular}{|c|c|c|c|c|c|c|c|c|c|c|c|}
\hline \multirow{3}{*}{$\begin{array}{l}\text { Factor } \\
\text { Resected }\end{array}$} & \multirow{3}{*}{$\begin{array}{l}\text { Group } \\
\text { Resected }\end{array}$} & \multirow{3}{*}{$\begin{array}{l}n \\
280\end{array}$} & \multirow{3}{*}{$\begin{array}{l}\text { Events } \\
37\end{array}$} & \multicolumn{4}{|c|}{ Univariate Cox regression } & \multicolumn{4}{|c|}{ Multivariate Cox regression } \\
\hline & & & & \multirow{3}{*}{$\begin{array}{l}p \\
0.11\end{array}$} & \multirow{2}{*}{$\begin{array}{l}\mathrm{HR} \\
1.00\end{array}$} & \multicolumn{2}{|c|}{$95 \% \mathrm{Cl}$ of $\mathrm{HR}$} & \multirow[t]{2}{*}{$\mathrm{p}$} & \multirow[t]{2}{*}{$\mathrm{HR}$} & \multicolumn{2}{|c|}{$95 \% \mathrm{Cl}$ of $\mathrm{HR}$} \\
\hline & & & & & & & & & & & \\
\hline & Unresected & 82 & 11 & & 1.75 & 0.88 & 3.47 & 0.06 & & & \\
\hline \multirow{3}{*}{$\begin{array}{l}\text { Site of primary } \\
\text { tumour }\end{array}$} & Right sided & 82 & 13 & & 1.00 & & & & & & \\
\hline & Residual colon & 148 & 16 & 0.05 & 0.47 & 0.22 & 1.00 & 0.08 & & & \\
\hline & Rectum & 130 & 19 & 0.42 & 0.75 & 0.37 & 1.52 & 0.35 & & & \\
\hline \multirow[t]{2}{*}{ Sites of metastases* } & Non-peri/oment & 295 & 34 & & 1.00 & & & & & & \\
\hline & Peri/oment & 67 & 14 & 0.007 & 2.35 & 1.26 & 4.40 & 0.04 & 1.98 & 1.03 & 3.82 \\
\hline Age & Continuous & 362 & 48 & 0.002 & 0.96 & 0.93 & 0.99 & 0.001 & 0.96 & 0.93 & 0.98 \\
\hline \multirow[t]{2}{*}{ Sex } & Male & 229 & 29 & & 1.00 & & & & & & \\
\hline & Female & 133 & 19 & 0.60 & 1.17 & 0.66 & 2.08 & 0.67 & & & \\
\hline CEA & Continuous & 346 & 47 & 0.31 & 1.00 & 1.00 & 1.00 & 0.36 & & & \\
\hline LDH & Continuous & 142 & 21 & 0.32 & 1.00 & 0.999 & 1.000 & - & & & \\
\hline $\mathrm{Hb}$ & Continuous & 362 & 48 & 0.07 & 0.82 & 0.67 & 1.02 & 0.43 & & & \\
\hline Alk phosph & Continuous & 359 & 48 & 0.46 & 1.00 & 0.999 & 1.002 & - & & & \\
\hline Albumin & Continuous & 359 & 48 & 0.42 & 0.97 & 0.91 & 1.04 & - & & & \\
\hline \multirow[t]{2}{*}{ PS category } & $0-1$ & 273 & 38 & & 1.00 & & & & & & \\
\hline & $2-4$ & 83 & 9 & 0.60 & 1.22 & 0.59 & 2.53 & 0.48 & & & \\
\hline
\end{tabular}

*Presence of absence of peritoneal and/or omental metastases (peri/oment).

HR, Hazard ratio; CEA, carcinoembryonic antigen; LDH, lactate dehydrogenase; Hb, haemoglobin; Alk phosph, alkaline phosphatase; PS, performance status.

The proportion of patients who were admitted as a result of major intestinal haemorrhage was similar in the unresected (3.7\%, 95\% CI $0.8-10.3 \%$ ) and resected $3.9 \%$, 95\% CI 1.7-6.2\%) groups. Multivariate Cox regression analysis showed that no factor was significantly associated with major gastrointestinal haemorrhage. However, a greater proportion of patients in the unresected group required transfusions of more than a total of five units of blood $(20.7 \%, 95 \%$ CI $12.6-31.1 \%)$, or received three or more transfusions $(14.6 \%$, $95 \%$ CI $7.8-24.2 \%)$, suggesting that they suffered more severe occult gastrointestinal bleeding. Multivariate logistic regression analysis showed that an unresected primary tumour (HR 2.28, 95\% CI 1.1-4.7; p=0.03), male sex (HR 0.45, 95\% CI $0.23-0.88 ; \mathrm{p}=0.02)$, and pretreatment haemoglobin (HR 0.66, 95\% CI 0.52-0.83; p <0.001)) were associated with increased transfusion requirements. The site of the primary tumour was not associated with increased transfusion requirements $(\mathrm{p}=0.84)$.

Fifteen patients $(18.3 \%, 95 \%$ CI $10.6-28.4 \%)$ in the unresected group received abdominal radiotherapy. Eleven (13.3\%) received radiotherapy to control local symptoms of pain and/or bleeding whereas four $(4.9 \%)$ received radiotherapy to control the primary tumour in the context of an excellent response at the sites of metastatic disease and/or limited metastatic disease. Twenty seven patients $(9.6 \%, 95 \%$ CI $6.2-13.1 \%$ ) in the resected group received abdominal radiotherapy; $24(8.6 \%)$ patients receiving radiotherapy for symptoms of pain and/or bleeding and three (1.1\%) patients for asymptomatic local relapse.

\section{Survival}

Median survival of patients in the unresected group was 8.2 months compared with 14.0 months for the resected group. Survival was not a primary end point of this study. However, a multivariate analysis was performed to determine whether the resection status of the primary tumour had a strong impact on the likelihood of death while controlling for the differences between groups. The most significant factors affecting survival were the presence of peritoneal or omental metastases (HR 1.49,95\% CI 1.10-2.00; $\mathrm{p}=0.01$ ), performance status (HR 1.43, 95\% CI 1.10-1.90; $\mathrm{p}=0.02$ ), serum alkaline phosphatase (HR 1.001,95\% CI 1.001-1.001; $p<0.001$ ), and serum albumin (HR 0.93, 95\% CI 0.90-0.96; $\mathrm{p}<0.001$ ). The resection status of the primary tumour was not significantly associated with survival $(\mathrm{p}=0.08)$.

\section{DISCUSSION}

Colorectal cancer is the second most common cause of cancer death in the Western world and approximately $25 \%$ of patients have metastatic disease at the time of presentation. ${ }^{12}$ A major goal of therapy in these patients is the maintenance of quality of life. The utility of any treatment must be carefully judged based on the effects of such therapy on tumour related symptoms and complications, as well as considering the results of the likely side effects of treatment on overall quality of life.

The main finding from this study was that the incidence of most major intestinal complications among patients with unresected primary colorectal cancer and synchronous metastases who received initial treatment with chemotherapy was low. In particular, the most serious complications of peritonitis and major gastrointestinal haemorrhage affected less than $4 \%$ of these patients. Similarly, intestinal obstruction affected only $13.4 \%$ of patients in the unresected group and its likelihood was most strongly associated with peritoneal or omental disease and age. While peritoneal and omental disease are known to be associated with obstruction, the reasons why age should have a significant effect are not clear.

Palliative surgery has frequently been advocated for these patients, both to treat symptoms associated with the primary tumour and to prevent major intestinal complications such as intestinal obstruction, perforation, or major haemorrhage. ${ }^{3}$ However, other investigators have recognised that patients with metastatic colorectal cancer who have undergone palliative resection of the primary tumour still face the prospect of further intestinal complications, which may require further surgery. ${ }^{4}$ This fact was also evident in our study where, for example, $13.2 \%$ of patients who had undergone a prior palliative resection developed subsequent episodes of intestinal obstruction, some of which required surgical intervention.

The postoperative morbidity and mortality associated with palliative surgery in patients with metastatic colorectal cancer is considerable, and is higher than that observed after curative resection. Representative series suggest that postoperative mortality after palliative surgery in these patients ranges from $4.6 \%$ to $10 \%$, with postoperative morbidity ranging from $23 \%$ to $50 \% .^{13}{ }^{14}$ Higher morbidity and mortality rates have been observed after palliative surgery in patients with a variety of different tumour types, and this has been partly attributed to an impaired nutritional status as a consequence of the underlying malignancy. ${ }^{15}$ Thus the routine use of palliative surgery may adversely affect both quality of life and survival of patients. 
Although there was no significant difference in the incidence of most major intestinal complications between the unresected and resected groups, this study suggested that prior palliative resection reduced the subsequent requirement for transfusion or radiotherapy. The requirement for transfusions is unlikely to significantly affect the quality of life as few patients $(14.6 \%)$ in the unresected group required three or more transfusions.

The low incidence of most major intestinal complications observed in the unresected group in this study therefore suggests that many patients presenting with colorectal carcinomatosis may be safely spared the potential morbidity and mortality of routine palliative surgery. It also suggests that the economic costs of surgery may be avoided in many cases.

Three previous reports involving smaller numbers of patients with metastatic colorectal cancer and unresected primary tumours, who received less uniform initial treatment, have reported low incidences of subsequent intestinal complications. ${ }^{13}{ }^{16}{ }^{17}$ Scoggins et al described the outcome of 23 patients who received initial treatment with chemotherapy alone, radiotherapy alone, or the combination of chemotherapy and radiotherapy. ${ }^{13}$ The incidence of intestinal obstruction was $8.7 \%(n=2)$ with no episodes of intestinal haemorrhage or perforation. The incidence of intestinal complications in patients with metastatic disease, who had previously undergone a palliative surgical procedure, was not reported in this study. Mahteme et al described the outcome of 70 patients with locally advanced or metastatic rectal cancer. ${ }^{16}$ However, over $50 \%$ of this group had undergone a palliative defunctioning procedure and only $11 \%$ of the group received chemotherapy. Surgery was required in $7 \%$ of patients with an unresected primary tumour for late intestinal complications. Crane et al have described the effects of chemoradiation on pelvic symptom control and the requirement for colostomy in 55 patients with primary rectal cancer and synchronous metastases. ${ }^{17}$ Five patients had a colostomy fashioned before commencing chemoradiation but in the remaining patients the one year actuarial colostomy free status was $87 \%$.

Two major recent developments in the treatment of patients with metastatic colorectal cancer provide a basis for a significant change in the management of patients with this disease. The first stems from the improvements made in chemotherapy for colorectal cancer over the past decade. Survival of patients with untreated metastatic colorectal cancer is poor and is principally limited by the presence of metastatic tumour. The typical median survival time for untreated patients with metastatic colorectal cancer is eight months ${ }^{18}$ and the most important factor predicting survival is the initial performance status. ${ }^{19} 20$ Chemotherapy has been shown to palliate symptoms attributable to metastatic disease as well as to achieve prolongation of survival in patients with metastatic colorectal cancer. $^{18}$ Previously, palliative chemotherapy was largely restricted to fluoropyrimidine drugs. However, new chemotherapy agents including irinotecan or oxaliplatin combined with 5-FU as the initial therapy have been shown to have significant activity in advanced colorectal cancer and may provide palliation and increase median overall survival times to approximately 17 months. ${ }^{1121}$

The early use of chemotherapy in metastatic colorectal cancer has been shown to be superior to delayed or expectant treatment. ${ }^{22}$ This is important because initial treatment with surgery will inevitably delay institution of palliative chemotherapy. As survival is principally limited by the presence of metastatic disease, the early use of chemotherapy represents a more logical approach to the treatment of disseminated tumour as it comprises a systemic rather than a local modality of therapy. This approach is particularly appropriate in patients who have limited macroscopic tumour dissemination, where initial treatment with chemotherapy provides valuable information about its sensitivity to chemotherapy while simultaneously treating microscopic metastases. This information can be used to allow the selection of appropriate candidates with limited metastatic disease for surgical intervention such as potentially curative metastasectomy, which is associated with long term survival in a proportion of cases. ${ }^{23}$

The second major development derives from increasing success in the management of intestinal obstruction or haemorrhage using endoscopic techniques. Thus tumours causing intestinal obstruction can be successfully palliated using stents inserted under fluoroscopic or colonoscopic guidance. When used as definitive palliative treatment, over $90 \%$ of stents remained patent after six months of follow up. ${ }^{24}$ Similarly, endoscopic ablative therapies may be useful in controlling both obstructive and haemorrhagic symptoms. These may include endoscopic laser therapy such as the Nd:YAG (neodymium yttrium aluminium garnet) laser, cryotherapy, transanal resection, or photodynamic therapy. ${ }^{25} \mathrm{Nd}$ :YAG laser therapy can provide effective palliation in almost $90 \%$ of colorectal cancer cases with complication rates of less than 10\% and with low treatment related mortality. ${ }^{26}$ As our study showed that most patients with an unresected primary tumour and synchronous metastases did not develop major intestinal complications, an approach using initial chemotherapy alone, reserving endoscopic therapies for those patients who actually develop specific complications due to their primary tumour, is likely to be a feasible approach.

A limitation of this study is its retrospective nature, which means that formal assessment of the effect of lack of initial surgical treatment of the primary tumour on both symptoms and overall quality of life could not be evaluated. Furthermore, it is impossible to determine whether non-randomised case selection may have affected the results. It is inevitable and appropriate that patients who had presented with symptoms or signs demanding urgent surgical management would have undergone initial surgery, and initial treatment with chemotherapy would not be advocated in these cases. It is also impossible to determine accurately in this retrospective analysis what effect surgical resection has on survival. The superior survival observed in the resected group is likely to reflect differences in tumour burden, as these patients had better prognostic parameters such as performance status, serum albumin, and alkaline phosphatase compared with the unresected group. ${ }^{27}{ }^{28}$ The two groups are not strictly comparable for survival as patients with rapidly progressive tumour, or patients who died or suffered significant morbidity related to surgery, would not subsequently proceed to receive chemotherapy. The multivariate analysis of survival, which controls for baseline differences between the groups, suggests that any impact of surgical resection of the primary tumour on survival is small.

The care of patients with metastatic colorectal cancer now usually occurs in a multidisciplinary setting. Surgery will remain an important component of the multidisciplinary care of patients with metastatic colorectal cancer. Approximately $20 \%$ of such patients require urgent surgical intervention at the time of diagnosis for complications such as obstruction or perforation, ${ }^{29}$ and selected patients may be candidates for metastasectomy. However, this study suggests that the majority of the remaining patients can be safely treated with chemotherapy, if this treatment is deemed appropriate, in the knowledge that the intestinal complication rate is low. Subsequent interventions for selected patients may include open surgery, endoscopic ablative therapy, stent insertion, or radiotherapy. The increasing complexity of the different possible approaches to the management of patients with metastatic colorectal cancer will require careful evaluation of their respective benefits. These should be prospectively analysed by comparing the effects of different interventions on symptoms, quality of life, survival, complications, and cost of care. 


\section{ACKNOWLEDGEMENT}

We are grateful to Mr T A Cook, Dr M Allen, and Dr I Chau for critica review of the manuscript.

\section{Authors' affiliations}

N C Tebbutt, A R Norman, D Cunningham, M E Hill, D Tait J Oates, S Livingston, J Andreyev, Gastrointestinal Unit, Royal Marsden Hospital, London and Surrey, UK

\section{REFERENCES}

1 McArdle CS, Hole D, Hansell D, et al. Prospective study of colorectal cancer in the west of Scotland: 10-year follow-up. Br J Surg 1990;77:280-2

2 Mella J, Biffin A, Radcliffe AG, et al. Population-based audit of colorectal cancer management in two UK health regions. Colorectal Cancer Working Group, Royal College of Surgeons of England Clinica Epidemiology and Audit Unit. Br J Surg 1997;84:1731-6.

3 Ellis $\mathbf{H}$. Curative and palliative surgery in advanced carcinoma of the large bowel. BM 1971; 3:291-3.

4 Makela J, Haukipuro K, Laitinen S, et al. Palliative operations for colorectal cancer. Dis Colon Rectum 1990;33:846-50.

5 Kaplan EL, Meier P. Non-parametric estimation from incomplete observations. J Am Stat Assoc 1958; 53:457-81.

6 Peto R, Peto J. Asymtotically efficient invariant procedures. J R Stat Soc 1972; 135: 185-206

7 Cox D, Oakes D. Analysis of Survival Data. New York: Chapman Hall, 1984.

8 Seymour MT, Slevin ML, Kerr DJ, et al. Randomized trial assessing the addition of interferon alpha-2a to fluorouracil and leucovorin in advanced colorectal cancer. Colorectal Cancer Working Party of the United Kingdom Medical Research Council. J Clin Oncol 1996; 14:2280-8

9 Ross $\mathbf{P}$, Norman A, Cunningham D, et al. A prospective randomised trial of protracted venous infusion 5-fluorouracil with or without mitomycin $C$ in advanced colorectal cancer. Ann Oncol 1997;8:995-1001.

10 Cunningham D, Zalcberg JR, Rath U, et al. Final results of a randomised trial comparing "Tomudex" (raltitrexed) with 5-fluorouracil plus leucovorin in advanced colorectal cancer. "Tomudex" Colorectal Cancer Study Group. Ann Oncol 1996;7:961-5.

11 Douillard JY, Cunningham D, Roth AD, et al. Irinotecan combined with fluorouracil compared with fluorouracil alone as first-line treatment for metastatic colorectal cancer: a multicentre randomised trial. Lance 2000;355: 1041-7.

12 Price T, Cunningham D, Hickish T, et al. Phase III study of mitomycin-C with protracted venous infusion 5 fluorouracil or circadian timed infusion 5 fluorouraci in advanced colorectal cancer. Proc Am Soc Clin Oncol 1999; 18:262a (abstract 1008).
13 Scoggins CR, Meszoely IM, Blanke CD, et al. Nonoperative management of primary colorectal cancer in patients with stage IV disease. Ann Surg Oncol 1999;6:651-7.

14 Joffe J, Gordon PH. Palliative resection for colorectal carcinoma. Dis Colon Rectum 1981;24:355-60.

15 Meguid MM, Debonis D, Meguid V, et al. Complications of abdominal operations for malignant disease. Am J Surg 1988;156:341-5.

16 Mahteme H, Pahlman L, Glimelius B, et al. Prognosis after surgery in patients with incurable rectal cancer: a population-based study. Br J Surg 1996:83:1116-20.

17 Crane CH, Janjan NA, Abbruzzese JL, et al. Effective pelvic symptom control using initial chemoradiation without colostomy in metastatic rectal cancer. Int J Radiat Oncol Biol Phys 2001;49:107-16.

18 Simmonds PC. Palliative chemotherapy for advanced colorectal cancer: systematic review and meta-analysis. Colorectal Cancer Collaborative Group. BM 2000;321:531-5.

19 Assersohn L, Norman A, Cunningham D, et al. Influence of metastatic site as an additional predictor for response and outcome in advanced colorectal carcinoma. Br J Cancer 1999;79:1800-5.

20 Lavin P, Mittelman A, Douglass H Jr, et al. Survival and response to chemotherapy for advanced colorectal adenocarcinoma: an Eastern Cooperative Oncology Group report. Cancer 1980;46:1536-43.

21 de Gramont A, Figer A, Seymour M et al. Leucovorin and fluorouracil with or without oxaliplatin as first-line treatment in advanced colorectal cancer. J Clin Oncol 2000;18:2938-47.

22 Nordic Gastrointestinal Tumor Adjuvant Therapy Group. Expectancy or primary chemotherapy in patients with advanced asymptomatic colorectal cancer: a randomized trial. J Clin Oncol 1992;10:904-11.

23 Fong $Y$, Fortner J, Sun RL, et al. Clinical score for predicting recurrence after hepatic resection for metastatic colorectal cancer: analysis of 1001 consecutive cases. Ann Surg 1999;230:309-18.

24 Camunez F, Echenagusia A, Simo G, et al. Malignant colorectal obstruction treated by means of self-expanding metallic stents: effectiveness before surgery and in palliation. Radiology 2000;216:492-7

25 Dohmoto M, Hunerbein M, Schlag PM. Palliative endoscopic therapy of rectal carcinoma. Eur J Cancer 1996;32A:25-9.

26 Mathus-Vliegen EM, Tytgat GN. Laser ablation and palliation in colorectal malignancy. Results of a multicenter inquiry. Gastrointest Endosc 1986;32:393-6.

27 Bedikian AY, Chen TT, Malahy MA, et al. Prognostic factors influencing survival of patients with advanced colorectal cancer: hepatic-artery infusion versus systemic intravenous chemotherapy for liver metastases. $J$ Clin Oncol 1984;2:174-80.

28 Steinberg J, Erlichman C, Gadalla T, et al. Prognostic factors in patients with metastatic colorectal cancer receiving 5 -fluorouracil and folinic acid. Eur J Cancer 1992;28A: 1817-20.

29 Park YJ, Park KJ, Park JG et al. Prognostic factors in 2230 Korean colorectal cancer patients: analysis of consecutively operated cases. World J Surg 1999;23:721-6. 